\title{
Dupilumab in the management of moderate-to- severe asthma: the data so far
}

\author{
This article was published in the following Dove Press journal: \\ Therapeutics and Clinical Risk Management \\ I September 2017 \\ Number of times this article has been viewed
}

\author{
Pilar Barranco' \\ Elsa Phillips-Angles ${ }^{2}$ \\ Javier Dominguez-Ortega' \\ Santiago Quirce' \\ 'Department of Allergy, Hospital \\ La Paz Institute for Health Research \\ (IdiPAZ), CIBER de Enfermedades \\ Respiratorias (CIBERES), Madrid, \\ Spain; '2Department of Allergy, \\ Hospital La Paz Institute for Health \\ Research (IdiPAZ), Madrid, Spain
}

Correspondence: Pilar Barranco Department of Allergy, Hospital La Paz Institute for Health Research (IdiPAZ), CIBER de Enfermedades Respiratorias (CIBERES), P. La Castellana 26I, 28046, Madrid, Spain

Tel +34 9l 7277144

Email mpbarranco@gmail.com

\begin{abstract}
Severe asthma constitutes illness in a relatively small proportion of all patients with asthma, but it is a major public health problem - with considerable effect on morbidity, mortality, as well as a high burden on health care resources. Regardless of effective treatments being widely available and the existence of treatment guidelines, a large population of severe asthma cases remain uncontrolled. Achieving and maintaining asthma control in this group of patients is, therefore, of utmost importance. The recognition of distinct inflammatory phenotypes within this population has driven the development of targeted biological therapies - particularly, selective targeted monoclonal antibodies (mAbs). It is noteworthy that in approximately $50 \%$ of these patients, there is strong evidence of the pathogenic role of Thelper type-2 (Th2) cytokines, such as interleukin (IL)-4 and IL-13, orchestrating the eosinophilic and allergic inflammatory processes. Among the recently developed antiasthma biologic drugs, the mAb dupilumab is very promising given its ability to inhibit the biological effects of both IL-4 and IL-13. In this review, we focused on IL-4 and IL-13, as these interleukins are considered to play a key role in the pathophysiology of asthma, and on dupilumab, an anti-IL-4 receptor human mAb, as a forthcoming treatment for uncontrolled severe asthma in the near future.
\end{abstract}

Keywords: dupilumab, asthma, interleukin-4, interleukin-13, monoclonal antibodies, treatment

\section{Introduction}

Asthma is a complex heterogeneous disease, with different pathogenic mechanisms, clinical presentations, and responses to treatment, usually characterized by chronic airway inflammation. ${ }^{1}$ Wheeze, shortness of breath, chest tightness and cough that vary over time and in intensity, together with variable expiratory airflow limitation, are the main asthma features. ${ }^{1}$ Asthma affects an estimated 241 million children and adults in the world, with a prevalence rate of $1 \%-18 \%$ of the general population in different countries. ${ }^{2}$ Approximately $5 \%-10 \%$ of the asthmatic population is affected with severe asthma, requiring high-dose inhaled corticosteroids (ICS) in addition to a second controller (and/or systemic corticosteroids) to prevent it from the disease becoming uncontrolled or for asthma which remains uncontrolled despite combination therapy. ${ }^{3}$ Even though severe asthma constitutes illness in a relatively small proportion of all patients with asthma, it is a major public health problem, with considerable impacts on morbidity, mortality, as well as a high burden within health care resources. ${ }^{4,5}$ Regardless of effective treatments being widely available and the existence of treatment guidelines, a large population of severe asthma cases remain uncontrolled. ${ }^{6}$ Achieving and maintaining better asthma control in this group of patients is, therefore, of utmost importance.

The recognition of distinct phenotypes within this population has driven the development of targeted biological therapies, particularly selective targeted monoclonal 
antibodies (mAbs). It is noteworthy that in approximately $50 \%$ of these patients, there is strong evidence of the pathogenic role of $\mathrm{T}$ helper type-2 (Th2) cytokines such as interleukin (IL)-4, IL-5, and IL-13 orchestrating the eosinophilic and allergic inflammatory processes. ${ }^{7}$ Biologics that are approved for pharmacological treatment of severe persistent asthma include omalizumab, mepolizumab, and reslizumab, but other mAbs under clinical development are expected to be approved soon. ${ }^{8}$

In this review, we focused on IL-4 and IL-13, as these interleukins are considered to play a key role in the pathophysiology of asthma, and on dupilumab, an anti-IL-4 receptor human $\mathrm{mAb}$, as a forthcoming treatment for uncontrolled asthma in the near future.

\section{Role of IL-4 and IL- I 3 in asthma pathobiology}

The pathophysiology of allergic asthma is typically orchestrated by type 2 lymphocytes, which organize the airways' immune inflammatory response. A wide range of interactions that take place between the innate and adaptive immune systems produce a Th2-high pattern. ${ }^{9}$ Aeroallergens invade the airway epithelium, thus stimulating Toll-like receptors that act in innate responses. Toll-like receptors activate a signaling cascade, increasing airway production of several cytokines, such as IL-25, thymic stromal lymphopoietin (TSLP), and IL-33, which activate Th2 adaptive responses.
Production of TSLP can also stimulate Th2 inflammation by polarizing dendritic cells that synthesize chemokines, such as CCL17 and CCL22, attracting Th2 lymphocytes that express a chemokine receptor (CCR4). ${ }^{10}$ Once these Th2 lymphocytes are carrying the CCR4 receptor, they produce a large amount of Th2-derived cytokines, such as IL-5, IL-9, IL-4, and IL-13, thus activating the development and chemotaxis of different cells implicated in immunoglobulin E ( $\mathrm{IgE})$-mediated asthma such as basophils, eosinophils, and mast cells. ${ }^{11}$

Both IL-4 and IL-13 are principally produced by type 2 innate lymphoid and CD4 ${ }^{+}$Th2 cells. However, basophils, mast cells, $\mathrm{CD}^{+}$Th cells, eosinophils, and natural killer (NK) cells can also secrete these cytokines. ${ }^{12,13}$ These cytokines are involved in the inflammatory and structural changes that take place in asthmatic airways (Figure 1). As shown in the figure, IL-4 and IL-13 promote Ig switching from class M to class E antibodies, at the level of B lymphocytes and plasma cells. ${ }^{14}$ Moreover, they facilitate airway recruitment of eosinophils by activating eotaxin synthesis and upregulation of endothelial adhesion molecules like the vascular cell adhesion molecule-1. The polarization and maintenance of Th2-type cells is an important role of IL-4. ${ }^{15}$ IL-13 stimulates airway epithelial expression of inducible nitric oxide synthase (iNOS) and mucus production. Furthermore, an important role of IL-13 is airway remodeling by enhancing goblet cell hyperplasia, promoting the transformation of bronchial fibroblasts into myofibroblasts, enhancing the contractile capacity of airway

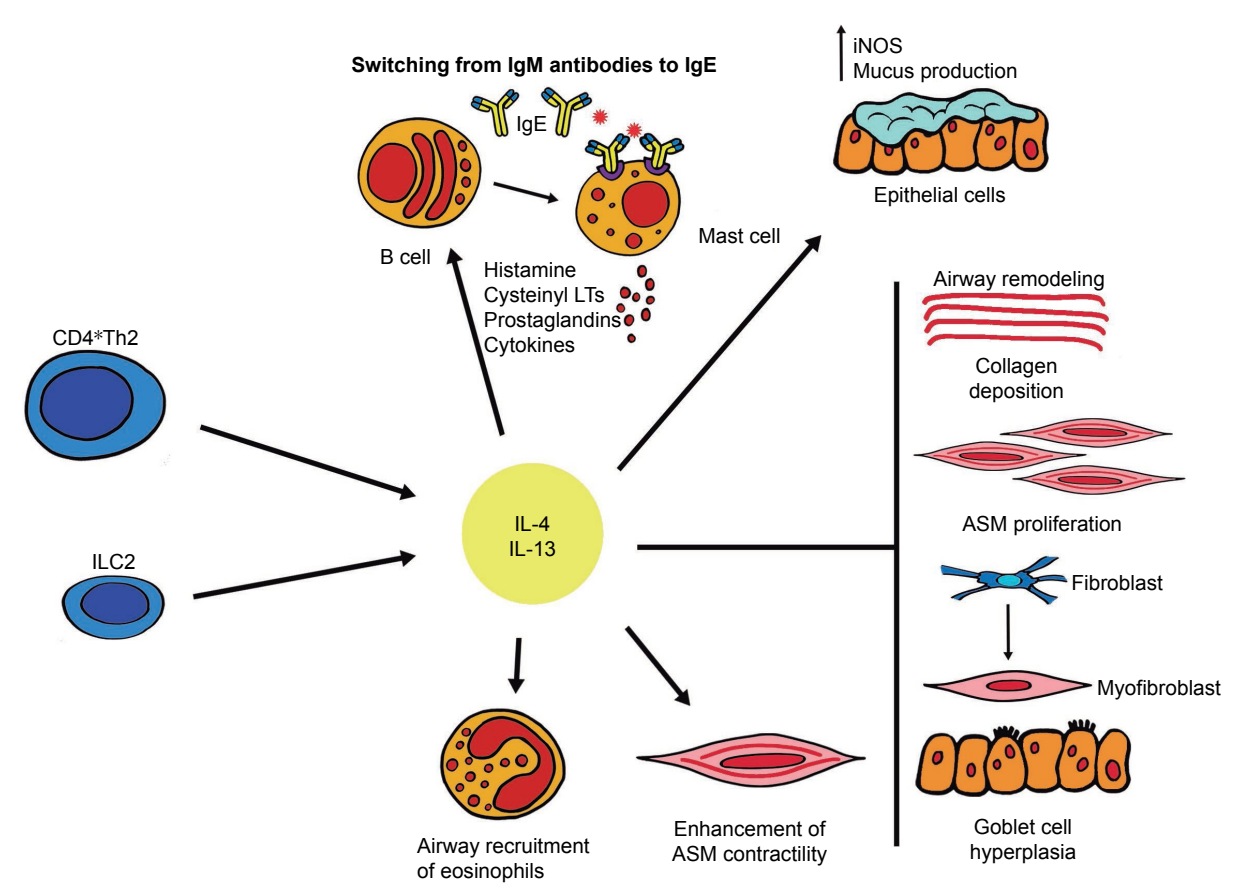

Figure I Inflammatory and structural changes in asthmatic airways promoted by IL-4 and IL-I3.

Abbreviations: ASM, airway smooth muscle cells; ILC2, type 2 innate lymphoid cells; iNOS, inducible nitric oxide synthase; LTs, leukotrienes. 
smooth muscle cells (ASM), and also enhancing extracellular deposition of collagen. ${ }^{13,16}$ Many inflammation-derived bronchoconstrictors such as histamine, leukotrienes, endothelin-1, or prostaglandin D3, to name a few, are overexpressed in subjects with asthma and likely add to ASM activation. ${ }^{16}$ All of these modifications induced by IL-4 and IL-13 lead to a noticeable increment in airway hyperresponsiveness.

These fundamental cytokines in airway hyperresponsiveness play their role in inducing the activation of a heterodimeric receptor complex comprising the IL-13 receptor $\alpha 1$ subunit (IL-13R $\alpha 1$ ) and the IL-4 receptor $\alpha$ subunit (IL-4R $\alpha$ ), ${ }^{17}$ as shown in Figure 2. They are expressed on B lymphocytes, eosinophils, dendritic cells, monocytes/ macrophages, basophils, bronchial epithelial cells, endothelial cells, fibroblasts, and ASM cells. The interaction of IL-4 and IL-13 with this receptor complex stimulates tyrosine kinase 2 (Tyk2) and Janus kinase 1/2 (JAK 1/2), constitutively associated with IL-4/IL-13 receptor chains. ${ }^{18,19}$ These two tyrosine kinase proteins, situated within the cytoplasm, are activated and this stimulates the cascade of phosphorylation-dependent activation and nuclear translocation of the signal transducer and activator of transcription 6 (STAT6). STAT6 is a transcription factor which regulates the expression of some genes that encode various molecules. These molecules act as key mediators of IL-13 and IL-4 biological effects. Moreover, Tyk 2 and JAK $1 / 2$ s have different signaling kinetics and potencies. IL-4's high-affinity binding to IL- $4 \mathrm{R} \alpha$ is independent of $\gamma$-chain or IL-13R $\alpha 1$ association, whereas the affinity of IL-13 binding to IL-13R $\alpha 1$ is increased by the presence of IL-4R $\alpha .{ }^{20}$ IL-4 can bind through two types of receptor complexes (type 1 and type 2) which are found on hematopoietic and non-hematopoietic cells, respectively. The binding of IL-4 to IL-4R $\alpha$, through the type 1 complex, induces the dimerization with $\gamma$ chain, activating JAK 1 and JAK 3 which are also responsible for the phosphorylation and subsequent stimulation of STAT6. On the other hand, when IL-13 binds to the IL-13 receptor $\alpha 2$ chain (IL-13R $\alpha 2$ ), its mechanism of action can be inhibited, given that this union does not cause a cascade of signaling, thereby producing an autoregulatory negative loop limiting the activity of IL-13. ${ }^{21}$

Asthmatic patients, in comparison with non-asthmatic controls, have high levels of IL-4 and IL-13 in induced sputum, serum, airway mucosa, and bronchoalveolar lavage fluid. ${ }^{22-25}$ Genetic research has shown that patients undergoing allergen challenges show significant airway increases of IL-4/IL-13 mRNAs and protein levels. ${ }^{23,26-28}$

Several studies have been carried out with knockout mice models, concluding that although IL-4 and IL-13 act together, they are sufficient individually to cause similar pathology. ${ }^{29-31}$

\section{Biomarkers}

Identifying the role of specific cytokines in the pathophysiology of asthma paved the way for defining the asthma

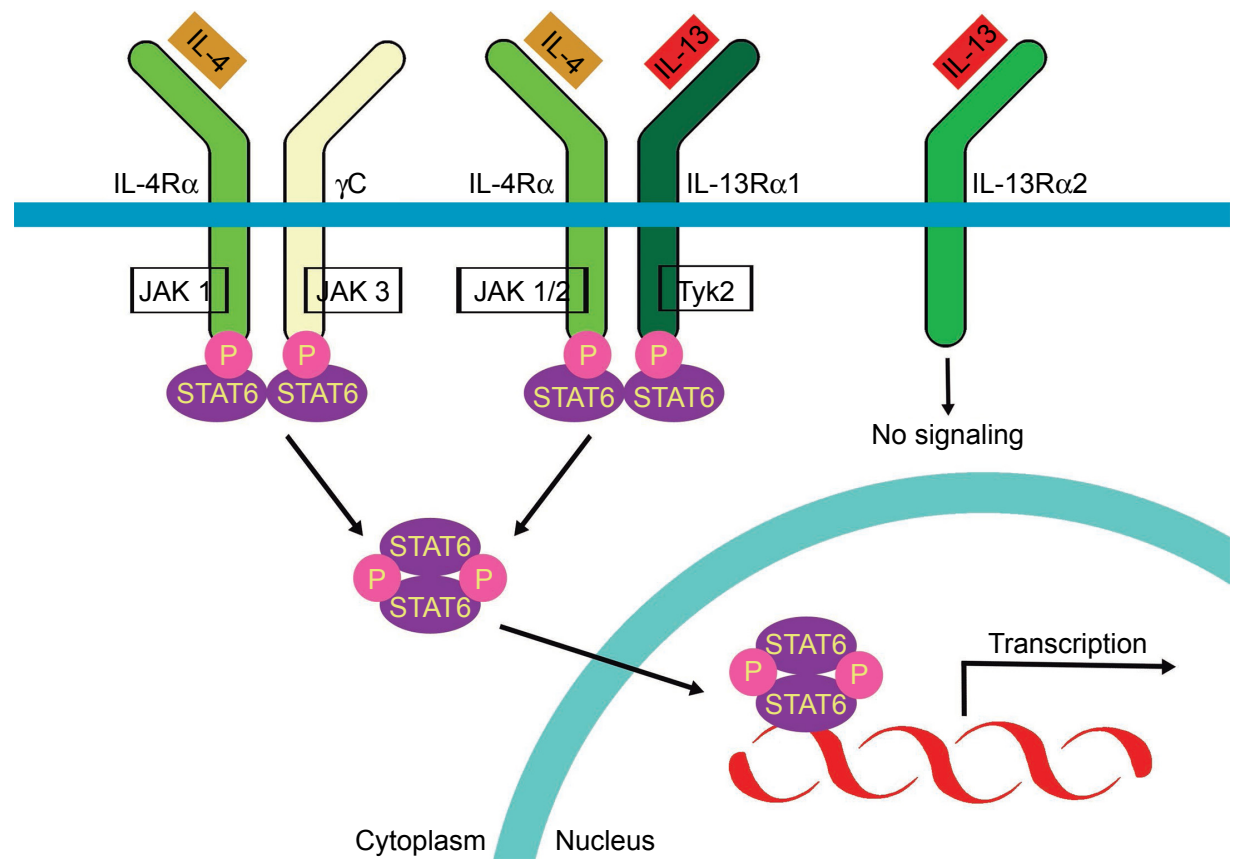

Figure 2 Activation of a heterodimeric receptor complex comprised of the IL-I3 receptor $\alpha$ I subunit (IL- I3R $\alpha$ I) and the IL-4 receptor $\alpha$ subunit (IL-4 $\alpha$ ) by IL- I 3 and IL-4. Abbreviations: IL, interleukin; JAK, Janus kinase; STAT, signal transducer and activator of transcription; Tyk, tyrosine kinase. 
phenotype. Specific biomarkers of Th2 airway inflammation such as total serum IgE, sputum and blood eosinophil count, serum levels of periostin and osteopontin, and fractional exhaled nitric oxide (FeNO) help classify asthma into different subtypes, by showing the predominant pathophysiological mechanism, ${ }^{32}$ helping predict future risk, ${ }^{33}$ and identifying patients for future Th2-targeted therapies.

Sputum and blood eosinophil counts were the first biomarkers to be identified and used to predict corticosteroid responsiveness. A study conducted by Woodruff et $\mathrm{al}^{9}$ reported that patients with elevated bronchial expression of IL-5 and IL-13 had higher levels of sputum and blood eosinophilia than non-asthmatic controls. In a different study carried out by Wenzel et al, ${ }^{34}$ sputum and blood eosinophils were used as type 2 biomarkers to identify subjects with eosinophilic moderate-to-severe asthma for a trial of dupilumab - an anti-IL-4 receptor- $\alpha$-subunit. No improvement in the levels of blood or sputum eosinophilia was found despite the decrease in other biomarkers such as FeNO and the improvement of $\mathrm{FEV}_{1}$. Given these results, it can be hypothesized that the eosinophilia associated with this molecular phenotype is not controlled by IL-4 or IL-13.

Both IL-13 and IL-4 regulate the synthesis of IgE, thus making it an important biomarker of Th2 cell activation. As a result of $\operatorname{IgE}$ binding with the high-affinity receptor (FceRI) found in basophils and mast cells, there is a cellular activation which ends in the liberation of various inflammatory mediators including cytokines such as IL-5, IL-4, and IL-13. However, some studies have stated that total $\mathrm{IgE}$ is not a good biomarker to predict eosinophilic inflammation. ${ }^{35}$

High levels of expression of IL-13- and IL-4-inducible genes, which include periostin, are characteristic of Th2 inflammation. ${ }^{36}$ Periostin is a matricellular protein which contributes to airway remodeling and its serum levels have been described as the best predictor of sputum eosinophilia when compared with other biomarkers. ${ }^{35}$ This biomarker was also reported to predict improvement in lung function after treatment with lebrikizumab, an anti-IL-13 mAb. ${ }^{37}$

Periostin, chloride channel accessory 1 (CLCA1), and Serpin $\beta 2$ are specifically upregulated by IL-13 in airway epithelial cells. While the mean sputum expression of IL-4, IL-5, and IL-13 was noted to correlate with the expression of periostin and CLCA1, it does not correlate with the sputum gene expression of Serpin $\beta 2$. They are thought to predict sputum and blood eosinophilia in patients with mild asthma, but this has yet to be determined in patients with more severe asthma. $^{38}$

Given that NO-synthase activity and NO production are promoted by IL-13, FeNO levels can be used as a biomarker of Th2 inflammation and of IL-13 levels in the bronchial mucosa. It has also been reported that FeNO levels are good predictors of steroid responsiveness, as patients with high levels of FeNO respond better to corticosteroid therapy than those with low levels. ${ }^{39}$ FeNO levels decrease following treatment with anti-IL-4/13 therapy. ${ }^{34,40}$

\section{Therapies targeting IL-4 and IL-I 3}

\section{Anti IL-I3}

Tralokinumab is a human Ig-G4 monoclonal antibody that potently and specifically neutralizes IL-13. Two Phase IIa studies have evaluated the effect of tralokinumab, with controversial results regarding asthma symptoms score and $\mathrm{FEV}_{1}$. Piper et al published a trial in which 194 subjects were randomized to receive tralokinumab $(150,300$, or $600 \mathrm{mg})$ or placebo subcutaneously every 2 weeks. ${ }^{41}$ Although tralokinumab treatment was associated with improved lung function, no improvement in Asthma Control Questionnaire (ACQ-6), the primary endpoint, was observed across the tralokinumab doses tested. In an subsequent Phase IIb trial, Brightling et $\mathrm{al}^{42}$ selected patients aged 18-75 years with severe asthma, and two to six exacerbations in the year prior to the study, to be randomly assigned (1:1) to tralokinumab in one of two dosing regimens, or placebo. A dose of $300 \mathrm{mg}$ given either every 2 weeks or every 4 weeks did not significantly reduce asthma exacerbation rates. However, $\mathrm{FEV}_{1}$ was significantly increased in patients receiving tralokinumab every 2 weeks whereas increases in $\mathrm{FEV}_{1}$ with tralokinumab given every 4 weeks did not differ significantly from placebo. Results of post hoc subgroup analyses showed improvements in pre-bronchodilator $\mathrm{FEV}_{1}$, ACQ-6, and quality of life questionnaires in a subgroup with a high level of serum dipeptidylpeptidase-4, compared to placebo. Moreover, improvements in asthma exacerbation rate, ACQ-6, and prebronchodilator $\mathrm{FEV}_{1}$ was found in subjects with periostin concentrations higher than the median, thus suggesting a possible treatment effect in a defined population of patients with severe uncontrolled asthma who had a combination of raised biomarker concentrations, had postbronchodilator reversibility, and were not receiving maintenance oral corticosteroids. Both tralokinumab regimens showed an acceptable safety and tolerability profile over the 52-week treatment period and during the 22-week follow-up period. There is currently an ongoing Phase III study (STRATOS2) evaluating the efficacy and safety of tralokinumab in adults and adolescents with uncontrolled asthma (NCT02194699). ${ }^{43}$

Anrukinzumab is a fully humanized $\mathrm{IgG}_{1}$ anti-IL-13 $\mathrm{mAb}$. It blocks the cytokine and prevents the activation of the receptor. Its pharmacokinetics have been studied in healthy 
volunteers and patients with different stages of asthma and ulcerative colitis, ${ }^{44}$ but clinical studies in asthma have since been discontinued.

IMA-638 (anrukinzumab) was also tested in a human allergen-inhalation challenge model. The study was a doubleblind, randomized, placebo-controlled trial with parallel groups in which 27 subjects were enrolled and completed the study. The subjects were nonsmokers, aged 18 to 60 years, with an $\mathrm{FEV}_{1}$ that was at least $70 \%$ of the predicted value, with provocative concentration of methacholine leading to a $20 \%$ fall in $\mathrm{FEV}_{1}$ of less than $16 \mathrm{mg} / \mathrm{mL}$. Responses to the inhaled allergen challenge were measured. The participants who developed an early-phase asthmatic response (a fall in $\mathrm{FEV}_{1}$ of at least $20 \%$ within $1 \mathrm{~h}$ after allergen challenge), followed by a late-phase asthmatic response (a fall of at least 15\% between 3 and $7 \mathrm{~h}$ after allergen challenge), were randomized to receive drug or placebo $(1: 1)$ which were administered as two subcutaneous doses (1 week apart). IMA-638 inhibited the early- and late-phase asthmatic response, whereas it showed no effect on airway hyperresponsiveness to methacholine, blood or sputum eosinophils, or total serum $\operatorname{IgE} .{ }^{45}$

Lebrikizumab is an IgG4 humanized mAb that specifically binds to IL-13, inhibiting its function. Corren et $\mathrm{al}^{37}$ conducted a randomized, double-blind, placebo-controlled study in which they randomized 219 patients with moderateto-severe persistent uncontrolled asthma to receive $250 \mathrm{mg}$ of subcutaneous lebrikizumab monthly for 6 months. At 12 weeks, there was a significant increase in baseline $\mathrm{FEV}_{1}$ of $5.5 \%$ in the active group, compared to the placebo group. There was no significant reduction in the number of exacerbations. However, among patients in the high-periostin subgroup on lebrikizumab, the increase from baseline $\mathrm{FEV}_{1}$ was $8.2 \%$ higher than in the placebo group ( $p=0.03$ ), with no significant differences among patients in the low-periostin subgroup. Hanania et $\mathrm{a}^{46}$ showed that the rate of exacerbations was $60 \%$ lower in periostin-high patients receiving lebrikizumab compared to those receiving placebo. This suggests that a group of patients selected by biomarkers might obtain a greater benefit from therapy with anti-IL-13 mAbs. However, in Phase III studies, lebrikizumab did not consistently show significant reductions in asthma exacerbations in patients with elevated biomarkers, and the clinical development of lebrikizumab for asthma treatment was halted. ${ }^{47}$

GSK67958 is a humanized mAb that inhibits IL-13 binding to both of the IL-13 receptors ( $\alpha 1$ and $\alpha 2)$. Although it was well tolerated, it did not demonstrate clinically significant improvements in asthma control, pulmonary function, or in exacerbations in patients with severe asthma. ${ }^{48}$

\section{Anti IL-4}

Pitrakinra is an IL-4R alpha antagonist. In two independent Phase IIa clinical trials in patients with atopic asthma, Wenzel et $\mathrm{al}^{49}$ demonstrated that a $25 \mathrm{mg}$ subcutaneous dose had no significant effect in maximum change in $\mathrm{FEV}_{1}$ after allergen challenge compared to placebo. There were no significant differences in exacerbation rates when comparing participants on active treatment with those on placebo. However, administration of $60 \mathrm{mg}$ nebulized pitrakinra was associated with a significantly lower average decrease in $\mathrm{FEV}_{1}$ percentage compared to placebo (4.4\% vs $15.9 \%)$ after allergen challenge. In a later pharmacogenetic study, Slager et $\mathrm{al}^{50}$ showed that in a non-Hispanic white population with moderateto-severe asthma and a common genotype (rs8832GG), treatment with pitrakinra could significantly reduce asthma exacerbations in a dose-dependent manner.

Developments of other anti-IL-4 receptor agents such as AMG-317 $17^{51}$ or pascolizumab ${ }^{52}$ have been discontinued.

\section{Dupilumab}

Dupilumab (REGN668/SAR231893; Regeneron/Sanofi Pharmaceuticals, Tarrytown, NY, USA and Paris, France) is a human IgG4 antibody ${ }^{53}$ anti-IL-4 receptor $\alpha$-subunit. Dupilumab blocks the transduction signal activated by IL-13 and IL-4 and has been evaluated in patients with persistent asthma (moderate-to-severe) and airway or peripheral eosinophilia. ${ }^{34,54}$ Following the report of successful trials with dupilumab in adults with moderate-tosevere atopic dermatitis (AD), ${ }^{55,56}$ its use was approved to treat the condition, thus confirming the importance of Th2 pathway activation in the majority of adults with moderate/severe AD.

\section{Clinical efficacy of dupilumab}

\section{Phase I studies}

Data on the structure, chemical, pharmacodynamic, pharmacokinetic properties, and metabolism of dupilumab are not available. Two Phase I studies with dupilumab were concluded in 2010 (NCT01015027, ${ }^{57}$ NCT01484600 58 ), and two studies were concluded in 2012 (NCT01537653, ${ }^{59}$ NCT01537640 60 in healthy volunteers evaluating tolerability, pharmacokinetics, and safety of subcutaneous or intravenous doses of dupilumab, but results are not available.

\section{Phase II studies}

A Phase IIa randomized, double-blind placebo-controlled parallel-group study was conducted to evaluate the safety, tolerability, and efficacy of SAR231893/REGN688 administrated subcutaneously once-weekly for 12 weeks in subjects 
with persistent eosinophilic moderate-to-severe asthma. It was carried out with participants whose asthma was uncontrolled or partially controlled with ICS at medium-tohigh doses (fluticasone $\geq 250 \mu \mathrm{g}$ or equivalent) plus a longacting beta ${ }_{2}$ ggonist (salmeterol $50 \mu \mathrm{g}$ or equivalent), twice daily. ${ }^{34}$ It took place from March 2011 to October 2012 at 28 sites in the United States. The selected subjects were 18 to 65 years old and had been diagnosed with asthma for at least 12 months, and had a high sputum eosinophil count ( $\geq 3 \%$ ) or a high blood eosinophil level ( $\geq 300$ cells $/ \mu L$ ). Other inclusion criteria were: having had at least one severe asthma exacerbation in the 2 years before the screening, having an $\mathrm{FEV}_{1} \geq 50 \%$ of the predicted value during the screening and randomization stages, and a score of 1.5 to 3.0 on the ACQ-5 during the screening. Subjects were randomly assigned in a 1:1 ratio to receive once-weekly subcutaneous injections of $300 \mathrm{mg}$ dupilumab or placebo for 12 weeks. Participants received 250 or $500 \mu \mathrm{g}$ of fluticasone and $50 \mu \mathrm{g}$ salmeterol twice daily (according to pre-trial dose) for 4 weeks. Subjects were then asked to suspend salmeterol at Week 4 and to suspend ICS from weeks 6 to 9. The main objective was to study the occurrence of an asthma exacerbation. An exacerbation was defined as: a reduction of $30 \%$ or more in morning peak expiration flow (PEF) from baseline on two consecutive days; at least six additional reliever inhalations of albuterol in a $24 \mathrm{~h}$ period relative to baseline on two consecutive days; an exacerbation of asthma requiring treatment with systemic corticosteroids; an increase in ICS of at least four times the most recent dose; or hospitalization due to an asthma exacerbation during the study period of 12 weeks.

The trial included a total of 104 subjects (dupilumab group: 52 subjects; placebo group: 52 subjects). Three participants $(6 \%)$ on dupilumab had an asthma exacerbation, compared to 23 (44\%) subjects on placebo - an $87 \%$ reduction. No subjects required hospitalization due to asthma exacerbations. The most frequent events considered to be an exacerbation of asthma were an increase in the use of reliever medication and a reduction in morning PEF, each reported in $2 \%$ and $19 \%$ of patients in the dupilumab and placebo groups, respectively. $\mathrm{FEV}_{1 \text { Aldo }}$ improved by more than $200 \mathrm{~mL}$ when dupilumab (as compared with placebo) was added to ICS and LABA. The increase was even sustained during their tapering and discontinuation.

The morning asthma-symptom scores, ACQ-5 scores, use of albuterol, and episodes of nocturnal awakenings improved more with dupilumab than with placebo. With dupilumab, the majority of patients had little or no change in peripheral blood eosinophil levels and, with placebo, no changes were observed.

Th2-associated biomarkers such as FeNO, eotaxin-3, serum IgE, and thymus and activation-regulated chemokine (TARC) decreased with dupilumab, confirming the biologic activity of dupilumab. ${ }^{40,61}$ The degree of improvement of $\mathrm{FEV}_{1}$ corresponded to the degree of reduction in the FeNO level. No significant changes were observed in YKL-40 or CEA levels between study groups.

These results are different from those observed in studies with other mAbs that target IL-13 exclusively. ${ }^{37,41}$ In a Phase II placebo-controlled study in adults with moderateto-severe asthma, tralokinumab improved lung function and decreased the use of beta-agonists, but no improvement was observed in asthma symptoms. ${ }^{41}$ In a randomized, double-blind, placebo-controlled study in adults who had asthma that was inadequately controlled despite inhaled glucocorticoid therapy, lebrikizumab was only associated with improvements in lung function. ${ }^{37}$

Later, a pivotal randomized, double-blind, placebocontrolled, dose-ranging, parallel-group Phase IIb clinical trial (NCT01854047) ${ }^{62}$ took place in 174 study sites across 16 countries. ${ }^{54}$ Participants met the inclusion criteria when aged $\geq 18$ years, had a physician diagnosis of moderate-tosevere uncontrolled asthma for $\geq 12$ months according to the Global Initiative for Asthma 2009 guidelines, and had received ICS at medium-to-high doses (fluticasone $\geq 250 \mu \mathrm{g}$ or equivalent) plus à long-acting beta ${ }_{2}$ agonist (salmeterol $50 \mu \mathrm{g}$ or equivalent), twice daily. Their $\mathrm{FEV}_{1}$ was between $40 \%$ and $80 \%$ of predicted values, and the Juniper Asthma Control Questionnaire 5-question version (ACQ-5) score was $\geq 1.5$. The subjects had to have experienced a hospitalization, emergency, or urgent care visit, or systemic corticosteroid treatment due to asthma worsening, in the previous year.

A total of 776 patients were randomly assigned (1:1:1:1:1) to receive subcutaneous dupilumab $200 \mathrm{mg}(\mathrm{n}=150)$ or $300 \mathrm{mg}(\mathrm{n}=157)$ every 2 weeks, $200 \mathrm{mg}(\mathrm{n}=154)$ or $300 \mathrm{mg}$ $(n=157)$ every 4 weeks, or placebo $(n=158)$, over a 24 -week period.

The primary outcome was the change from baseline in $\mathrm{FEV}_{1}$ in subjects with baseline blood eosinophil levels of at least $300 / \mu \mathrm{L}$, assessed in the intention-to-treat population at Week 12. In the group of subjects with eosinophil levels of at least $300 / \mu \mathrm{L}(\mathrm{n}=325)$, all dupilumab dose regimens (except for the $200 \mathrm{mg}$ every 4 weeks dose regimen) were associated with significant increases in $\mathrm{FEV}_{1}$ from baseline to Week 12 which ranged from 0.35 to $0.43 \mathrm{~L}$ and from 0.17 to $0.26 \mathrm{~L}$ 
versus placebo. Increases were maintained throughout the 24-week treatment period and were significant for all dose regimens (except for $200 \mathrm{mg}$ dupilumab every 4 weeks).

Secondary endpoints were pre-specified at Weeks 12 and 24 for both the overall population and for the subgroup with eosinophil counts of at least $300 / \mu \mathrm{L}$. These included percentage change from baseline in $\mathrm{FEV}_{1}$, time to severe exacerbation events during treatment, annualized severe asthma exacerbation rate, and change in morning and evening asthma symptom scores, ACQ-5 score, number of inhalations per day of salbutamol for symptom relief, and the Asthma Quality of Life Questionnaire (AQLQ) score. Changes from baseline in FeNO levels at weeks 12 and 24 were also assessed. There was an assessment regarding safety outcomes in all patients who had received at least part of a dose of dupilumab.

With regard to secondary outcomes, treatment with dupilumab administered every 2 weeks was associated with reduced risk of the annualized severe exacerbation event rate estimate in the overall population in asthmatic patients with eosinophil counts $\geq 300 / \mu \mathrm{L}$, and in asthmatic patients with eosinophil counts $<300 / \mu \mathrm{L}$.

In the subgroup with eosinophil counts $\geq 300 / \mu \mathrm{L}$ and in the overall population, improvements in ACQ- 5 total scores at Week 24, relative to baseline, were significantly greater in subjects who received dupilumab every 2 weeks than in those who received placebo. In the subgroup with eosinophil levels of $<300 / \mu \mathrm{L}$, a significant improvement in ACQ-5 score was found only for the subgroup receiving $200 \mathrm{mg}$ dupilumab every 2 weeks in comparison to those who had received placebo.

In the overall population, the global AQLQ scores at Week 24 were significantly higher than the baseline score in subjects on dupilumab every 2 and 4 weeks than in subjects on placebo, with the exception of those receiving $200 \mathrm{mg}$ dupilumab every 4 weeks. In the subgroup with eosinophil levels $\geq 300 / \mu \mathrm{L}$, the global AQLQ scores relative to baseline were significantly greater for all dose regimens of dupilumab compared with placebo.

All dupilumab dose regimens in the overall population showed significant dose-dependent reductions in FeNO at Week 24, with near-maximum decreases observed at Week 2.

Decreases in FeNO were higher for dupilumab administered every 2 weeks, and were maintained throughout treatment. In the subgroup with eosinophil counts $\geq 300 / \mu \mathrm{L}$, significant decreases in FeNO were observed at Week 24 (200 mg every 4 weeks, $p=0.0404 ; 300 \mathrm{mg}$ every 4 weeks, $p=0.0196$; $200 \mathrm{mg}$ every 2 weeks, $p<0.0001$; and $300 \mathrm{mg}$ every 2 weeks, $p<0.0001$ ), with differences ranging from $-23.40 \%$ to $-46.96 \%$ compared with placebo. Similar significant decreases in $\mathrm{FeNO}$ were found for the subgroup with eosinophil counts $<300 / \mu \mathrm{L}$ who received dupilumab every 2 weeks. Of the two doses of dupilumab given every 4 weeks, only the $300 \mathrm{mg}$ dose produced a significant decrease in FeNO compared with placebo $(p=0.004)$.

The efficacy of dupilumab (SAR231893/REGN668) in the treatment of bilateral nasal polyposis by assessment of the endoscopic nasal polyp score in comparison to placebo was evaluated in a Phase II, randomized, double-blind, placebo-controlled parallel group study (NCT01920893). ${ }^{63,64}$ The addition of subcutaneous dupilumab to mometasone furoate spray, compared with mometasone alone, reduced endoscopic nasal polyp burden after 16 weeks and the levels of total serum IgE, plasma eotaxin-3, and serum TARC. ${ }^{64}$

In a subgroup of 35 subjects with concomitant asthma (asthma diagnosis was based on patient history), treatment with dupilumab improved the absolute $\mathrm{FEV}_{1}, \mathrm{FEV}_{1}$ percentage of predicted value, and ACQ-5 score, when compared with the control group. ${ }^{64}$

\section{Safety and tolerability}

The published data shows that dupilumab is generally well tolerated. ${ }^{34,54}$

In the Phase IIa study, the most common adverse reactions were injection-site reactions, nasopharyngitis, nausea, and headache, all of which occurred more frequently with dupilumab than with placebo. One patient developed a progressive papular rash, urticaria, and edema which led to early discontinuation of dupilumab. ${ }^{34}$ Similar adverse reactions occurred in the Phase IIb study. A dose-response relationship with injection-site reactions was suggested. ${ }^{54}$

An unexpected transient elevation of blood eosinophils was observed in subjects with higher baseline serum eosinophil levels, and one patient developed a hypereosinophilic syndrome. Dupilumab was hence discontinued, and the patient rapidly responded to corticosteroid treatment. Two theories have been proposed for this finding of increased eosinophilia in response to dupilumab. First, the eosinophilia could represent a transient rebound elevation in response to eosinophil-promoting mediators such as IL-5, as a result of blocking IL-4 and IL-13. ${ }^{65}$ Second, as IL-4 and IL-13 recruit and facilitate eosinophil migration into tissues, the inhibition of these cytokines may cause eosinophil accumulation in the peripheral blood. ${ }^{66}$

In the clinical trial NCT01920893, ${ }^{63,64}$ the most commonly reported adverse events were nasopharyngitis (33\% in 
the placebo group vs $47 \%$ in the dupilumab group), injectionsite reactions ( $7 \%$ vs $40 \%$, respectively), and headaches $(17 \%$ vs 20\%). Transient increases in blood eosinophil levels were also reported in some patients after the initiation of treatment with dupilumab.

Until this issue is further explored, caution is advised for patients with highly elevated baseline eosinophil counts.

\section{Current clinical trials}

Clinical trials with dupilumab in patients with asthma are currently under development:

- Evaluation of dupilumab's effects on airway inflammation in patients with asthma (EXPEDITION; NCT02573233) ${ }^{67}$ is an exploratory, 24-week, randomized, double-blind, placebo-controlled, Phase II study carried out to evaluate the effects of dupilumab, administered every 2 weeks for 12 weeks, as add-on therapy to ICS/LABA, compared to placebo, on airway inflammation of adults with persistent asthma. The primary outcomes are the change from baseline of the number of inflammatory cells in the bronchial submucosa per square millimeter and the change from baseline of mucin-stained area in the bronchial submucosa per square millimeter. The secondary outcomes are the absolute change from baseline in exhaled NO levels and the proportion of patients with anti-drug antibodies who develop adverse reactions to dupilumab within 24 weeks.

- Evaluation of dupilumab in children with uncontrolled asthma (Lyberty Asthma Voyage; NCT02948959) ${ }^{68}$ is a randomized, double-blind, placebo-controlled, parallelgroup, Phase III study evaluating the efficacy and safety of dupilumab in children 6 to $<12$ years of age with uncontrolled persistent asthma. The primary outcome measure is the annual rate of severe exacerbations during the treatment period. The total study duration will be of up to 69 weeks per patient, including 3-5 weeks of screening, a randomized treatment period of 52 weeks, and a posttreatment observation period of 12 weeks.

- Evaluation of dupilumab in patients with persistent asthma (Lyberty Asthma Quest; NCT02414854) ${ }^{69}$ is a randomized, double-blind, placebo-controlled, parallelgroup study in Phase III evaluating the efficacy and safety of dupilumab in patients with persistent asthma. The primary outcomes are the annual rate of severe exacerbations during the treatment period ( 52 weeks) and the absolute change from baseline in pre-bronchodilator $\mathrm{FEV}_{1}$ (Week 12). The total study duration is 67-69 weeks per patient, including a screening period of $3-5$ weeks, treatment period of 52 weeks, and a posttreatment period of 12 weeks.

This clinical trial excludes patients with serum eosinophil levels $>1,500 / \mu \mathrm{L}$.

- Evaluation of dupilumab in patients with severe steroiddependent asthma (VENTURE; NCT02528214) ${ }^{70}$ is a randomized, double-blind, placebo-controlled Phase III study evaluating the efficacy and safety of dupilumab compared with placebo, in the reduction of maintenance oral corticosteroids in patients $\geq 12$ years of age with severe steroid-dependent asthma. The total study duration will be of up to 46 weeks per patient, including a screening period of 3-8 weeks, a randomized treatment period of 24 weeks, as well as a posttreatment observation period of 12 weeks.

- Long-Term safety evaluation of dupilumab in patients with asthma (Liberty Asthma Traverse; NCT02134028) ${ }^{71}$ is an open-label extension study that evaluates tolerability and long-term safety of dupilumab in patients ( $\geq 12$ years) with asthma, who had participated in previous dupilumab asthma clinical studies (DRI12544, PDY14192, EFC13579, or EFC13691). The primary endpoint of this study is the prevalence of patients experiencing any adverse events while undergoing treatment with dupilumab.

- Dupilumab compassionate use study (NCT03020810) ${ }^{72}$ is being undertaken to determine dupilumab's efficacy in subjects with extremely severe asthma, and to allow early access to a potentially effective therapy for very severe asthma patients ( $\geq 18$ years), who have been treated with several immunosuppressive drugs. The patients will receive a $600 \mathrm{mg}$ subcutaneous dupilumab loading dose on Day 1, and then $300 \mathrm{mg}$ subcutaneous dupilumab every 2 weeks. The study will be continued indefinitely, safety will be evaluated, and serious adverse events will be reported.

Other Phase III studies are also currently under development, for nasal polyposis (NCT0289845473), AD in adults (NCT01949311, ${ }^{74}$ NCT02277769, ${ }^{75}$ NCT02277743, ${ }^{76}$ NCT02260986, ${ }^{77}$ NCT02755649, ${ }^{78}$ and NCT0239513379) and children $\left(\mathrm{NCT} 02612454^{80}\right)$, and eosinophilic esophagitis (NCT02379052 ${ }^{81}$ ) in adults.

\section{Conclusion}

These studies show that dupilumab improves lung function and reduces the estimated exacerbation rate in patients with persistent asthma insufficiently controlled with medium-tohigh doses of ICS plus a LABA, irrespective of their baseline 
blood eosinophilic count. In adults with nasal polyposis refractory to intranasal corticosteroids and symptomatic chronic sinusitis, adding dupilumab to mometasone furoate nasal spray reduces endoscopic nasal polyp burden.

Dupilumab has also been reported to be effective in patients with $\mathrm{AD} .{ }^{55,56}$

Treatment with dupilumab is potentially useful in patients with severe asthma and associated comorbidities (AD, chronic sinusitis, and nasal polyposis) who remain inadequately controlled on standard therapy.

A potentially useful biomarker might be FeNO, as the enzymatic activity of NOS is directly controlled by both IL-4 and IL-13. A sustained reduction of $40 \%$ in FeNO levels during treatment with dupilumab has been found.

Phase III studies should provide answers about the efficacy of dupilumab, particularly with regard to the reduction of maintenance corticosteroid therapy, as patients on maintenance treatment with oral corticosteroids represent up to $46 \%$ of patients with severe asthma. ${ }^{82}$ These studies should also assess the efficacy and safety of dupilumab in children with severe asthma. Additional larger studies are required to establish the long-term safety and tolerability of dupilumab and to evaluate the rise of blood eosinophil levels observed in patients with asthma and elevated baseline serum eosinophilia.

Finally, Phase III studies of dupilumab should provide indications for anti-IL-4 receptor- $\alpha$ therapy and direct comparison with other medications.

\section{Acknowledgments}

The authors would like to thank Isabel Coman, native English-speaking MD, for her technical and grammatical assistance.

\section{Disclosure}

The authors report no conflicts of interest in this work.

\section{References}

1. Global Initiative for Asthma. GINA. [homepage on the Internet]. Available from: www.ginasthma.org. Accessed June 7, 2017.

2. Global Burden of Disease Study 2013 Collaborators. Global, regional, and national incidence, prevalence, and years lived with disability for 301 acute and chronic diseases and injuries in 188 countries, 1990-2013: a systematic analysis for the Global Burden of Disease Study 2013. Lancet. 2015;386(9995):743-800.

3. Chung KF, Wenzel SE, Brozek JL, et al. International ERS/ATS guidelines on definition, evaluation and treatment of severe asthma. Eur Respir J. 2014;43(2):343-373.

4. Sullivan PW, Campbell JD, Ghushchyan VH, Globe G. Outcomes before and after treatment after escalation to Global Initiative for Asthma steps 4 and 5 in severe asthma. Ann Allergy Asthma Immunol. 2015; 114(6):462-469.
5. Accordini S, Corsico AG, Braggion M, et al. The cost of persistent asthma in Europe: an international population-based study in adults. Int Arch Allergy Immunol. 2013;160(1):93-101.

6. Porsbjerg C, Menzies-Gow A. Co-morbidities in severe asthma: clinical impact and management. Respirology. 2017;22(4):651-661.

7. Bagnasco D, Ferrando M, Varricchi G, Passalacqua G, Canonica GW. A critical evaluation of anti-IL-13 and anti-IL-4 strategies in severe asthma. Int Arch Allergy Immunol. 2016;170(2):122-131.

8. Quirce S, Phillips-Angles E, Dominguez-Ortega J, Barranco P. Biologics in the treatment of severe asthma. Allergo Immunopathol. In press 2017.

9. Woodruff PG, Modrek B, Choy DF, et al. T-helper type 2-driven inflammation defines major sub-phenotypes of asthma. Am J Respir Crit Care Med. 2009;180(5):388-395.

10. Liu YJ. Thymic stromal lymphopoietin: master switch for allergic inflammation. J Exp Med. 2006;203(2):269-273.

11. Larché M, Robinson DS, Kay AB. The role of T lymphocytes in the pathogenesis of asthma. J Allergy Clin Immunol. 2003;111(3):450-463.

12. Steinke JW, Borish L. Th2 cytokines and asthma. Interleukin 4: its role in the pathogenesis of asthma, and targeting it for asthma treatment with interleukin-4 receptor antagonists. Respir Res. 2001;2(2):66-70.

13. Corren J. Role of interleukin-13 in asthma. Curr Allergy Asthma Rep. 2013;13(5):415-420.

14. Barnes PJ. The cytokine network in asthma and chronic obstructive pulmonary disease. J Clin Invest. 2008;118(11):3546-3556.

15. Paul WE, Zhu J. How are $\mathrm{T}(\mathrm{H}) 2$-type immune responses initiated and amplified? Nat Rev Immunol. 2010;10(4):225-235.

16. Auger L, Mailhot-Larouche S, Tremblay F, Poirier M, Farah C, Bossé Y. The contractile lability of smooth muscle in asthmatic airway hyperresponsiveness. Expert Rev Respir Med. 2016;10(1):19-27.

17. Oh CK, Geba GP, Molfino N. Investigational therapeutics targeting the IL-4/IL-13/STAT-6 pathway for the treatment of asthma. Eur Respir Rev. 2010;19(115):46-54.

18. Andrews R, Rosa L, Daines M, Khurana HG. Reconstitution of a functional human type II IL-4/IL-13 in mouse B cells: demonstration of species specificity. J Immunol. 2001;166(3):1716-1722.

19. Chiba Y, Goto K, Misawa M. Interleukin-13-induced activation of signal transducer and activator of transcription 6 is mediated by an activation of Janus kinase 1 in cultured human bronchial smooth muscle cells. Pharmacol Rep. 2012;64(2):454-458.

20. LaPorte SL, Juo ZS, Vaclavikova J, et al. Molecular and structural basis of cytokine receptor pleiotropy in the interleukin-4/13 system. Cell. 2008;132(2):259-272.

21. Zheng T, Liu W, Oh SY, et al. IL-13 receptor $\alpha 2$ selectively inhibits IL-13-induced responses in the murine lung. J Immunol. 2008;180(1): 522-529.

22. Vatrella A, Fabozzi I, Calabrese C, Maselli R, Pelaia G. Dupilumab: a novel treatment for asthma. J Asthma Allergy. 2014;7:123-130.

23. Maes T, Joos GF, Brusselle GG. Targeting IL-4 in asthma: lost in translation? Am J Respir Cell Mol Biol. 2012;47(3):261-270.

24. Daher S, Santos LM, Sole D, De Lima MG, Naspitz CK, Musatti CC. Interleukin-4 and soluble CD23 serum levels in asthmatic atopic children. J Investig Allergol Clin Immunol. 1995;5(5):251-254.

25. Walker C, Bauer W, Braun RK, et al. Activated T cells and cytokines in bronchoalveolar lavages from patients with various lung diseases associated with eosinophilia. Am J Respir Crit Care Med. 1994;150(4): $1038-1048$.

26. Corren J. Anti-interleukin-13 antibody therapy for asthma: one step closer. Eur Respir J. 2013;41(2):255-256.

27. Prieto J, Lensmar C, Roquet AI, et al. Increased interleukin-13 mRNA expression in bronchoalveolar lavage cells of atopic patients with mild asthma after repeated low-dose allergen provocations. Respir Med. 2000;94(8):806-814.

28. Zhu Z, Homer RJ, Wang Z, et al. Pulmonary expression of interleukin-13 causes inflammation, mucus hypersecretion, subepithelial fibrosis, physiologic abnormalities, and eotaxin production. J Clin Invest. 1999; 103(6):779-788 
29. Zheng T, Oh MH, Oh SY, Schroeder JT, Glick AB, Zhu Z. Transgenic expression of interleukin-13 in the skin induces a pruritic dermatitis and skin remodeling. J Invest Dermatol. 2009;129(3):742-751.

30. Rankin JA, Picarella DE, Geba GP, et al. Phenotypic and physiologic characterization of transgenic mice expressing interleukin 4 in the lung: lymphocytic and eosinophilic inflammation without airway hyperreactivity. Proc Natl Acad Sci U S A. 1996;93(15):7821-7825.

31. Chan LS, Robinson N, Xu L. Expression of interleukin-4 in the epidermis of transgenic mice results in a pruritic inflammatory skin disease: an experimental animal model to study atopic dermatitis. $J$ Invest Dermatol. 2001;117(4):977-983.

32. Bartminski G, Crossley M, Turcanu V. Novel biomarkers for asthma stratification and personalized therapy. Expert Rev Mol Diagn. 2015; 15(3):415-430.

33. Muñoz X, Bustamante V, Lopez-Campos JL, Cruz MJ, Barreiro E. Usefulness of noninvasive methods for the study of bronchial inflammation in the control of patients with asthma. Int Arch Allergy Immunol. 2015;166(1):1-12.

34. Wenzel S, Ford L, Pearlman D, et al. Dupilumab in persistent asthma with elevated eosinophil levels. N Engl J Med. 2013;368(26):2455-2466.

35. Jia G, Erickson RW, Choy DF, et al; Bronchoscopic Exploratory Research Study of Biomarkers in Corticosteroid-refractory Asthma (BOBCAT) Study Group. Periostin is a systemic biomarker of eosinophilic airway inflammation in asthmatic patients. J Allergy Clin Immunol. 2012;130(3):647-654.

36. Takayama G, Arima K, Kanaji T, et al. Periostin: a novel component of subepithelial fibrosis of bronchial asthma downstream of IL-4 and IL-13 signals. J Allergy Clin Immunol. 2006;118(1):98-104.

37. Corren J, Lemanske RF, Hanania NA, et al. Lebrikizumab treatment in adults with asthma. $N$ Engl J Med. 2011;365(12):1088-1098.

38. Peters MC, Mekonnen ZK, Yuan S, Bhakta NR, Woodruff PG, Fahy JV. Measures of gene expression in sputum cells can identify T2-high and T2-low subtypes of asthma. J Allergy Clin Immunol. 2014;133(2): 388-394.

39. Smith AD, Cowan JO, Brassett KP, et al. Exhaled nitric oxide: a predictor of steroid response. Am J Respir Crit Care Med. 2005;172(4): 453-459.

40. Chibana K, Trudeau JB, Mustovich AT, et al. IL-13 induced increases in nitrite levels are primarily driven by increases in inducible nitric oxide synthase compared with effects on arginases in human primary bronchial epithelial cells. Clin Exp Allergy. 2008;38(6):936-946.

41. Piper E, Brightling C, Niven R, et al. A phase II placebo-controlled study of tralokinumab in moderate-to-severe asthma. Eur Respir J. 2013; 41(2):330-338.

42. Brightling CE, Chanez P, Leigh R, et al. Efficacy and safety of tralokinumab in patients with severe uncontrolled asthma: a randomized, double-blind, placebo-controlled, phase $2 \mathrm{~b}$ trial. Lancet Respir Med. 2015;3(9):692-701.

43. AstraZeneca. A phase 3 study to evaluate the efficacy and safety of tralokinumab in adults and adolescents with uncontrolled asthma (STRATOS2). Available from: https://clinicaltrials.gov/ct2/show/ NCT02194699. NLM identifier: NCT02194699. Accessed April 24, 2017.

44. Hua F, Ribbing J, Reinisch W, Cataldi F, Martin S. A pharmacokinetic comparison of anrukinzumab, an anti-IL-13 monoclonal antibody, among healthy volunteers, asthma and ulcerative colitis patients. $\mathrm{Br} J$ Clin Pharmacol. 2015;80(1):101-109.

45. Gauvreau GM, Boulet LP, Cockcroft DW, et al. Effects of interleukin-13 blockade on allergen-induced airway responses in mild atopic asthma. Am J Respir Crit Care Med. 2011;183(8):1007-1014.

46. Hanania NA, Noonan M, Corren J, et al. Lebrikizumab in moderate-tosevere asthma: pooled data from two randomized placebo-controlled studies. Thorax. 2015;70(8):748-756.

47. Hanania NA, Korenblat P, Chapman KR, et al. Efficacy and safety of lebrikizumab in patients with uncontrolled asthma (LAVOLTA I and LAVOLTA II): replicate, phase 3, randomised, double-blind, placebocontrolled trials. Lancet Respir Med. 2016;4(10):781-796.
48. De Boever EH, Ashman C, Cahn AP, et al. Efficacy and safety of an anti-IL-13 mAb in patients with severe asthma: a randomized trial. J Allergy Clin Immunol. 2014;133(4):989-996.

49. Wenzel S, Wilbraham D, Fuller R, Getz EB, Longphre M. Effect of an interleukin-4 variant on late phase asthmatic response to allergen challenge in asthmatic patients: results of two phase 2a studies. Lancet. 2007;370(9596):1422-1431.

50. Slager RE, Otulana BA, Hawkins GA, et al. IL- 4 receptor polymorphisms predict reduction in asthma exacerbations during response to an anti-IL-4 receptor alpha antagonist. J Allergy Clin Immunol. 2012; 130(2):516-522.

51. Corren J, Busse W, Meltzer EO, et al. A randomized, controlled, phase 2 study of AMG 317, an IL-4Ralpha antagonist, in patients with asthma. Am J Respir Crit Care Med. 2010;181(8):788-796.

52. Hart TK, Blackburn MN, Brigham-Burke M, et al. Preclinical efficacy and safety of pascolizumab (SB 2406683): a humanized antiinterleukin-4 antibody with therapeutic potential in asthma. Clin Exp Immunol. 2002;130(1):93-100.

53. Reichert JM. Antibodies to watch in 2015. MAbs. 2015;7(1):1-8.

54. Wenzel S, Castro M, Corren J, et al. Dupilumab efficacy and safety in adults with uncontrolled persistent asthma despite use medium-to-highdose inhaled corticosteroids plus a long-acting $\beta_{2}$ agonist: a randomised double-blind placebo-controlled pivotal phase $2 \mathrm{~b}$ dose-ranging trial. Lancet. 2016;388(10039):31-44.

55. Beeck LA, Thaci D, Hamilton JD, et al. Dupilumab treatment in adults with moderate-to severe atopic dermatitis. N Engl J Med. 2014; 371(2):130-139.

56. Thaci D, Simpson EL, Beck LA, et al. Efficacy and safety of dupilumab in adults with moderate-to-severe atopic dermatitis inadequately controlled by topical treatments: a randomized, placebo-controlled, dose-ranging phase $2 b$ trial. Lancet. 2016;387(10013):40-52.

57. Regeneron Pharmaceuticals. Ascending dose study of the safety and tolerability of REGN668 (SAR231893) in normal healthy volunteers. Available from: https://clinicaltrials.gov/ct2/show/NCT01015027. NLM identifier: NCT01015027. Accessed May 18, 2017.

58. Regeneron Pharmaceuticals. Study of the safety, tolerability, pharmacokinetics, and immunogenicity of REGN668 administered subcutaneously to healthy volunteers. Available from: https://clinicaltrials.gov/ ct2/show/NCT01484600. NLM identifier: NCT01484600. Accessed May 18, 2017.

59. Sanofi. Safety, tolerability and pharmacokinetics of SAR231893 (REGN668) in healthy Japanese adult male subjects. Available from: https://clinicaltrials.gov/ct2/show/NCT01537653. NLM identifier: NCT01537653. Accessed May 18, 2017.

60. Sanofi. Comparison of the pharmacokinetics and safety of two SAR231893 (REGN668) drug products in healthy subjects. Available from: https://clinicaltrials.gov/ct2/show/NCT01537640. NLM identifier: NCT01537640. Accessed May 18, 2017.

61. Andrew DP, Chang MS, McNinch J, et al. STCP-1 (MDC) CC chemokine acts specifically on chronically activated Th2 lymphocytes and is produced by monocytes on stimulation with Th2 cytokines IL-4 and IL-13. J Immunol. 1998;161(9):5027-5038.

62. Sanofi. An evaluation of dupilumab in patients with moderate to severe uncontrolled asthma. Available from: https://clinicaltrials.gov/ct2/ show/NCT01854047?term=NCT01854047\&rank=1. NLM identifier: NCT01854047. Accessed June 7, 2017.

63. Sanofi. An evaluation of dupilumab in patients with nasal polyposis and chronic symptoms of sinusitis. Available from: https://clinicaltrials. gov/ct2/show/NCT01920893?term=NCT01920893\&rank=1. NLM identifier: NCT01920893. Accessed June 7, 2017.

64. Bachert C, Mannent L, Naclerio RM, et al. Effect of subcutaneous dupilumab on nasal polyp burden in patients with chronic sinusitis and nasal polyposis: a randomized clinical trial. JAMA. 2016;315(5): 469-479.

65. Chung KF. Dupilumab: a potential new treatment for severe asthma. Lancet. 2016;388(10039):3-4. 
66. Darveaux J, Busse WW. Biologics in asthma - the next step toward personalized treatment. J Allergy Clin Immunol Pract. 2015;3(2): $152-160$.

67. Sanofi. Evaluation of dupilumab's effects on airway inflammation in patients with asthma (EXPEDITION). Available from: https:// clinicaltrials.gov/ct2/show/NCT02573233. NLM identifier: NCT02573233. Accessed May 18, 2017.

68. Sanofi. Evaluation of dupilumab in children with uncontrolled asthma (VOYAGE). Available from: https://clinicaltrials.gov/ct2/show/ NCT02948959. NLM identifier: NCT02948959. Accessed May 18, 2017.

69. Sanofi. Evaluation of dupilumab in patients with persistent asthma (Liberty asthma quest). Available from: https://clinicaltrials.gov/ ct2/show/NCT02414854. NLM identifier: NCT02414854. Accessed May 18, 2017.

70. Sanofi. Evaluation of dupilumab in patients with severe steroid dependent asthma (VENTURE). Available from: https://clinicaltrials.gov/ ct2/show/NCT02528214. NLM identifier: NCT02528214. Accessed May 18, 2017.

71. Sanofi. Long-Term Safety Evaluation of dupilumab in patients with asthma (Liberty asthma traverse). Available from: https://clinicaltrials. gov/ct2/show/NCT02134028. NLM identifier: NCT02134028. Accessed May 18, 2017.

72. Wenzel SE. Dupilumab compassionate use study. Available from: https:/clinicaltrials.gov/ct2/show/NCT03020810. NLM identifier: NCT03020810. Accessed May 18, 2017.

73. Sanofi. Controlled clinical study of dupilumab in patients with nasal polyps (SINUS-52). Available from: https://clinicaltrials.gov/ct2/ show/NCT02898454. NLM identifier: NCT02898454. Accessed May 18, 2017.

74. Regeneron Pharmaceuticals. Open-label study of dupilumab (REGN668/ SAR231893) in patients with atopic dermatitis. Available from: https://clinicaltrials.gov/ct2/show/NCT01949311. NLM identifier: NCT01949311. Accessed May 18, 2017.

75. Regeneron Pharmaceuticals. Study of dupilumab (REGN668/ SAR231893) monotherapy administered to adult patients with moderate-to-severe atopic dermatitis (SOLO 2). Available from: https:// clinicaltrials.gov/ct2/show/NCT02277769. NLM identifier: NCT02277769. Accessed May 18, 2017.
76. Regeneron Pharmaceuticals. Study of dupilumab (REGN668/ SAR231893) monotherapy administered to adult patients with moderate-to-severe atopic dermatitis (SOLO 1). Available from: https:// clinicaltrials.gov/ct2/show/NCT02277743. NLM identifier: NCT02277743. Accessed May 18, 2017.

77. Regeneron Pharmaceuticals. Study to assess the efficacy and longterm safety of dupilumab (REGN668/SAR231893) in adult patients with moderate-to-severe atopic dermatitis. Available from: https:/clinicaltrials.gov/ct2/show/NCT02260986. NLM identifier: NCT02260986. Accessed May 18, 2017.

78. Regeneron Pharmaceuticals. A study to assess the efficacy and safety of dupilumab in patients with severe atopic dermatitis (AD) that are not controlled with oral cyclosporine A (CSA) or for those who cannot take oral CSA because it is not medically advisable. Available from: https:/clinicaltrials.gov/ct2/show/NCT02755649. NLM identifier: NCT02755649. Accessed May 18, 2017.

79. Regeneron Pharmaceuticals. A study to confirm the efficacy and safety of different dupilumab dose regimens in adults with atopic dermatitis (AD) (SOLO-continue). Available from: https://clinicaltrials.gov/ ct2/show/NCT02395133. NLM identifier: NCT02395133. Accessed May 18, 2017.

80. Regeneron Pharmaceuticals. A study to assess the long-term safety of dupilumab (REGN668/SAR231893) administered in patients 6 to $<18$ year of age with atopic dermatitis (AD). Available from: https:/clinicaltrials.gov/ct2/show/NCT02612454. NLM identifier: NCT02612454. Accessed May 18, 2017.

81. Regeneron Pharmaceuticals. Study of dupilumab in adult patients with active eosinophilic esophagitis (EoE). Available from: https:/clinicaltrials.gov/ct2/show/NCT02379052. NLM identifier: NCT02379052. Accessed May 18, 2017.

82. Shaw DE, Sousa AR, Fowler SJ, et al; U-BIOPRED Study Group. Clinical and inflammatory characteristic of the European U-BIOPRED adult severe asthma cohort. Eur Respir J. 2015;46(5):1308-1321.
Therapeutics and Clinical Risk Management

\section{Publish your work in this journal}

Therapeutics and Clinical Risk Management is an international, peerreviewed journal of clinical therapeutics and risk management, focusing on concise rapid reporting of clinical studies in all therapeutic areas, outcomes, safety, and programs for the effective, safe, and sustained use of medicines. This journal is indexed on PubMed Central, CAS,

\section{Dovepress}

EMBase, Scopus and the Elsevier Bibliographic databases. The manuscript management system is completely online and includes a very quick and fair peer-review system, which is all easy to use. Visit http://www.dovepress.com/testimonials.php to read real quotes from published authors. 